\title{
La reforma de las entidades territoriales: ¿por qué?'
}

\author{
Gérard Marcou \\ Profesor de la Universidad Paris 1 Panthéon-Sorbonne \\ (Escuela de Derecho), \\ Director del GRALE
}

Recibido: 30 de agosto de 2011 Aceptado: 24 de octubre de 2011

\begin{abstract}
La ley de 16 de diciembre de 2010, según la exposición de motivos se trataba de la "segunda etapa de la modernización de las estructuras territoriales de Francia", después de la reforma del "Estado territorial" emprendida en el 2007 en el marco de la Revisión General de las Políticas Públicas (RGPP), que refuerza la posición del nivel regional. Aunque en la elaboración de la ley se planteaba como objetivo la racionalización de la organización territorial, la realidad es que lo que se pretendía era la reducción de los gastos públicos incluidos los de las entidades territoriales. Pero como se señala en el artículo ni la reforma territorial ha finalizado ni el éxito de su puesta en marcha está garantizado.
\end{abstract}

Palabras claves Reforma territorial, gasto público, cooperación intermunicipal, fusiones de municipios, metrópolis, competencias.

\section{La réforme des collectivités territoriales: porquoi?}

\begin{abstract}
The law of 16 December 2010, according to the explanatory statement was the "second stage of modernization of french territorial structures", after reform of the "territorial State" undertaken in 2007 under the General Review of Public Policies (RGPP), which strengthens the position of the regional level. Although in the drafting of the law be raised aimed at the rationalization of territorial organization, the reality is that what was intended was the reduction of public expenditures, including those of the territorial entities. But as noted in the article nor the territorial reform has been completed or the success of their implementation underway is guaranteed.
\end{abstract}

Key words

Territorial reform, public expenditure, intermunicipal cooperation, merger of municipalities, metropolis, powers.

1 Este artículo ha sido publicado anteriormente en la revista Cahiers Français en su número de mayo-junio de 2011. La Revista de Estudios de la Administración Local y Autonómica ha obtenido la oportuna autorización de Cahiers Français para publicar. La traducción española de la versión original francesa ha sido realizada por Carmen Pineda Nebot. 
La reforma en la cual se inscribe la ley de 16 de diciembre de 2010 corresponde a un cambio de estrategia en la cúspide del Estado. Recordemos que el presidente Sarkozy, poco después de su elección, había pedido al ministro del Interior de entonces, Michèle Alliot-Marie, en su carta de objetivos de 30 de julio de 2007, hacer "una pausa en la concesión de nuevas competencias a las entidades territoriales, salvo excepciones de simplificación o de experimentación" y buscar los medios para una clarificación de las competencias. Sin embargo, desde el otoño de 2008, cuando el Ministerio del Interior estaba comprometido en la vía que le había sido indicada, el Presidente retomaba el tema y se comprometía, esta vez, en la vía de la reforma territorial, es decir de una reforma sobre las divisiones administrativas del territorio. Puso en marcha mediante el decreto del 22 de octubre de 2008 un "Comité para la reforma de las entidades territoriales", bajo la presidencia de Édouard Balladur cuyo informe fue publicado en marzo de 2009.

El informe Balladur trazaba la nueva orientación: es preciso en primer lugar simplificar las estructuras si se quiere clarificar la distribución de competencias². Sus propuestas se resumen así: generalizar la intermunicipalidad, reagrupar las regiones y los departamentos por los electos encargados de su administración, instituir "metrópolis" en algunas grandes aglomeraciones urbanas, reformar las finanzas locales especialmente con la supresión de la tasa profesional y el mantenimiento de una fiscalidad que vincule la empresa al territorio. Pero no incluía, en cambio, más que pocas propuestas concernientes a las competencias, más allá de una gran especialización de las diferentes categorías de las entidades territoriales.

El diagnóstico sobre el cual reposa la reforma está expresado en la exposición de motivos del proyecto de ley de reforma de las entidades territoriales: "La descentralización está esencialmente focalizada sobre las transferencias de competencias pero no ha modificado las estructuras (... ). El resultado es un paisaje institucional fragmentado (...). A la parcelación de las estructuras se añade el enmarañamiento de las competencias. (...). Resulta una pérdida de eficacia (...), un coste elevado para el contribuyente, una falta de inteligibilidad (... )"3. Se reconocía la inspiración de la "revisión general de las políticas públicas" (RGPP), que había ya dado lugar al informe Lambert. Éste se basaba en las relaciones entre el Estado y las entidades territoriales; preconizaba especialmente el reforzamiento de la intermunicipalidad y la especialización de las competencias de los departamentos y las regiones ${ }^{4}$. Según la exposición de motivos, se trataba de la "segunda etapa de la modernización de las estructuras territoriales de Francia”, después de la reforma del "Estado territorial" emprendida en el 2007 en el marco de la RGPP, y que refuerza la posición del nivel regional.

2 Comité para la reforma de las entidades locales, /l est temps de décider, Informe al Presidente de la República, Paris, La Documentation française, 2009.

3 Documento Senado, sesión ord. 2009-2010, n 64, 21 octubre de 2009.

4 Les relations entre l'État et les collectivités locales, informe del grupo de trabajo presidido por 56 Alain Lambert, RGPP, diciembre de 2007, Paris, La Documentation française, 2007. 
Detrás de la reforma en marcha se distingue un objetivo distinto al de la racionalización de la organización territorial, el de reducir los gastos públicos reduciendo también los de las entidades territoriales. Según el ponente de la comisión de las leyes de la Asamblea Nacional, Dominique Perben, "un número excesivo de estructuras territoriales con las competencias mal definidas debilita (...) la situación de nuestras finanzas públicas y la competitividad de nuestra economía". Señala que entre 2001 y 2008 la progresión de los gastos de funcionamiento de las entidades territoriales ha sido dos veces más rápida que la de los gastos de inversión $(54,5 \%$ frente a $25 \%$ ) y la progresión anual de los gastos públicos locales, excluyendo el peso de las transferencias de competencias del Estado, ha sido constantemente superior a la de la riqueza nacional5. La reforma de las estructuras y la regulación de las competencias se dirigen así a reducir los gastos públicos locales; se suma a la reforma de las finanzas locales, marcada especialmente por la supresión de la tasa profesional por la ley de presupuestos para 2010. La dimensión presupuestaria subyace en el conjunto de la reforma.

\section{LA REFORMA MUNICIPAL}

Francia se caracteriza por una extrema fragmentación municipal: hoy hay aún 36.500 municipios (communes) en el territorio metropolitano, de los que 14.400 tienen menos de 300 habitantes y sólo 900 municipios cuentan con más de 10.000 habitantes. Los inconvenientes de esta fragmentación han sido compensados, desde finales del siglo XIX, por el desarrollo de la cooperación intermunicipal, la red territorial de servicios del Estado y el recurso al sector privado para el desarrollo de los servicios urbanos. Es por la generalización de la intermunicipalidad y la perspectiva de nuevos municipios por lo que la ley de 16 de diciembre de 2010 trata de resolver este problema.

Los municipios y los departamentos nacieron de la Revolución francesa. Según la bonita definición dada por la Constitución de 1791, los municipios nacen de las "relaciones locales entre los ciudadanos". Pero el nivel de las relaciones locales no es hoy el de la Francia rural de finales del siglo XVIII. Sin embargo, el número de municipios, establecido en 38.000 por Napoleón Bonaparte, ha quedado más o menos estable hasta nuestros días, a pesar de una ligera disminución desde los años cincuenta. Por causa de este origen, el referente del municipio está en Francia en el municipio rural no en la ciudad, a diferencia de otros países europeos.

Después del fracaso de numerosas reformas tendentes a promover la fusión o el reagrupamiento de municipios, la más importante reforma de los últimos años ha sido, más aún que la revisión constitucional de 2003, la ley Chevènement de 1999 que

5 D. Perben, Rapport de la commission des lois sur le projet de loi de réforme des collectivités territoriales, Asamblea Nacional. $n^{\circ}$ 2516, mayo 2010, tomo 1, pp. 26-27. 
ha permitido, en algunos años, realizar lo esencial de la reforma territorial mediante la constitución de intermunicipalidades con fiscalidad propia ${ }^{6}$. A 1 de enero de 2011, 2.599 EPCI (Entidades Públicas de Cooperación Intermunicipal) con fiscalidad propia agrupaban a 35.041 municipios (95,5\%) y al 89,9\% de la población; la mitad de estas $\mathrm{EPCl}$ estaban sometidas al régimen de la fiscalidad profesional única y agrupaban a 47,6 millones de habitantes ${ }^{7}$. Un paso decisivo ha sido dado así, aunque 1.639 municipios (6,6 millones de habitantes) hayan quedado aislados. Se observa el establecimiento de una administración municipal a dos niveles, como ya por otra parte había sido considerado en 1968 por el plan Fouchet, jamás adoptado a causa de los "acontecimientos" del mismo año, antes del fracaso de la ley Marcellin de 1971 sobre la fusión de municipios.

\section{LA RACIONALIZACIÓN DE LA INTERMUNICIPALIDAD...}

De lo que se trata no es pues principalmente aquí de la reforma territorial -en lo esencial está llevada a cabo-, sino de racionalizar la intermunicipalidad y permitir su superación en la formación de municipios nuevos. El desarrollo de las intermunicipalidades (en términos jurídicos: entidades públicas de cooperación intermunicipal con fiscalidad propia) plantea tres tipos de problemas $^{8}$.

Se trata en primer lugar de un problema de legitimidad democrática, ya que los consejos están compuestos por delegados de los municipios elegidos por los consejos y no por sufragio directo, pese a tener el ejercicio de un poder fiscal y competencias ampliadas. La ley de 16 de diciembre de 2010 introduce la elección por sufragio directo, pero los miembros de los consejos siguen siendo los delegados de los municipios elegidos de la lista del consejo municipal en las elecciones municipales. Por otra parte, la elección directa no vale más que para los municipios que tienen una elección por sistema de lista, es decir los municipios de 3.500 habitantes o más, mientras que aproximadamente 20 millones de habitantes viven en alguno de los 33.500 municipios de menos de 3.500 habitantes.

Existe además un problema de estructura. Numerosas comunidades de municipios son muy pequeñas (126 tienen menos de 2.000 habitantes), muchas intermunici-

6 Sobre la aplicación de esta reforma, ver: "La réforme de l'intercommunalité", colección de estudios en: Annuaire zooo des Collectivités locales, CNRS Éditions, y especialmente: G. Marcou, “L’application de la loi du 12 juillet 1999. Stratégies et problèmes de mise en oeuvre", pp. 57-82.

7 Fuente: Ministerio del Interior, Dirección general de las entidades locales: http://manage.dgcl. interieur.gouv.fr/workspaces/members/des//documents/ intercommunalite/bilan_statistique/2011/bilan_ statistiqu_au/download-File/file/BilanStat_EPClaFP_Janv2011.pdf?nocache $=1296641877.0$

8 Véase, en particular:Tribunal de Cuentaš, L'intercommunalité en France, Informe al Presidente de la República, noviembre de 2005; Ph. Dallier, Rapport d'information fait au nom de l'Observatoire de la décentralisation, sur l'intercommunalité à fiscalité propre, Documento Senado, $n^{\circ} 193,1$ febrero de 2006; 58 J.-L. Albert, J. Fialaire, V. de Briant (dir.) (2008), L'intercommunalité et son coût, Paris, L'Harmattan, coll. 
palidades tienen perímetros insuficientes en relación al desarrollo urbano o demasiado dependientes de las oportunidades electorales. El número de sindicatos de municipios, forma tradicional de cooperación de servicios, no ha disminuido. No sólo desbordan los límites de las intermunicipalidades sino que la frecuente inadecuación de los perímetros de las intermunicipalidades en relación con ciertas funciones (planificación urbana, transportes públicos) ha conducido a la creación de nuevos sindicatos mixtos; algunos sindicatos responden a lógicas técnicas que cuadran mal con las lógicas territoriales de las intermunicipalidades (por ejemplo, para el agua o las comunicaciones electrónicas).

Por último, es necesario superar problemas de organización y de gestión que han tenido, sin ninguna duda, efectos inflacionistas. Según la ley, cuando un municipio está integrado en una intermunicipalidad, no puede intervenir directamente en las competencias transferidas y debe transferir al EPCI los servicios y los funcionarios que estaban encargados de ejercerlas. Estas reglas están lejos de ser respetadas: los municipios continúan interviniendo bajo el título de otras competencias; conservan una parte de los servicios y de los agentes y desarrollan nuevas actividades con estos medios humanos. Más allá de estas prácticas, la intermunicipalidad revela duplicidades que demandan una mayor mutualización de los medios. Por otro lado la EPCI debe reclutar personal para hacer frente a sus tareas, lo que conduce a un aumento injustificado de efectivos empleados. Sobre el plan financiero no hay que olvidar que el poder en las intermunicipalidades está estructurado en torno a los alcaldes de los municipios miembros. Esta circunstancia favorece una definición bastante generosa de las dotaciones de garantía y de solidaridad en beneficio de los municipios. Puede así suceder que la $\mathrm{EPCl}$ sea relativamente infrafinanciada en relación a los municipios miembros.

\section{... Y LAS GRANDES AGLOMERACIONES}

La ley de 16 de diciembre de 2010 introduce dos instituciones nuevas para tratar los problemas organizativos de las grandes aglomeraciones: la metrópoli y el polo metropolitano. La ley de 3 de junio de 2010 pone en marcha un dispositivo específico para encargarse de las necesidades de equipamiento del Gran Paris bajo la dirección del Estado. La uniformidad del estatuto municipal, que es la regla en la mayor parte de los países europeos, plantea dificultades cuando las desigualdades de tamaño y capacidad son tan importantes como en Francia. Recordemos que el artículo 89 de la Constitución de 1946 disponía ya que leyes orgánicas podrían "prever, para algunas grandes ciudades, reglas de funcionamiento y estructuras diferentes de las de los pequeños municipios e incluir disposiciones especiales para ciertos departamentos...". Pero es solamente con la creación de las comunidades urbanas, por la ley de 31 de diciembre de 1966 y con la política de las metrópolis de equilibrio introducida por la DATAR (Délégation à l'Aménagement du Territoire et à l'Action Régionale) en la misma época, cuando esta orientación ha encontrado un comienzo de 
realización`. La mundialización, y su corolario en la organización del espacio, la metropolización, se han convertido en apuestas mayores que requieren a la vez respuestas institucionales y respuestas en términos de equipamiento y urbanismo.

A falta de consenso sobre una respuesta institucional para el Gran Paris, el Estado responde con la recentralización de los poderes ${ }^{10}$, pero para las metrópolis la ley no sigue enteramente la propuesta del Informe Balladur, que era hacer una nueva entidad sustituyendo sobre su territorio no sólo a los municipios sino también al departamento. La metrópoli de la ley de 16 de diciembre de 2010 es de hecho una EPCI con fiscalidad propia, una supercomunidad urbana que toma ciertas competencias del departamento y, en menor medida, de la región, pero el departamento y la región conservan otras competencias sobre su territorio. Sólo el futuro dirá si los electos locales se apropian de la nueva institución y crean metrópolis en las aglomeraciones que cumplan las condiciones. En cuanto al polo metropolitano, da cuerpo a la idea de las redes de ciudades, que la DATAR había sostenido en los años 80; se tratará esta vez de un sindicato mixto asociando las intermunicipalidades de las aglomeraciones vecinas para unir sus proyectos de ordenación y desarrollo.

La inseguridad sobre el porvenir de los "nuevos municipios" es aún muy grande. Se trata de hecho de un nuevo procedimiento de fusión de municipios. La transformación de una intermunicipalidad en nuevo municipio debería ser un horizonte que expresara la culminación de un proceso de integración institucional y sociológica, en relación con la nueva escala de las relaciones locales. Sin embargo, las nuevas reglas que regulan el proceso de fusión pueden de hecho hacerlo más difícil, ya que el referéndum sobre el proyecto de fusión debe obtener la mayoría absoluta de los sufragios emitidos, representando al menos la cuarta parte de los electores inscritos en cada uno de los municipios afectados.

\section{LOS NIVELES INTERMEDIOS}

\section{El debate departamento-región}

¿Departamento y región? ¿Región o departamento? El debate no es nuevo, no está cerrado y nunca ha desembocado hasta hoy en una solución viable. La supresión del departamento parecía políticamente imposible, la de la región retrograda y por tanto el mantenimiento de estos dos niveles parece a la vez anacrónico y costoso, en medios y procedimientos, y las reformas sucesivas incluso han reforzado el departamento mediante la extensión y el peso presupuestario de las competencias que le han sido transferidas.

9 J.-P. Laborie, J.-F. Langumier, P. de Roo, La politique française d'aménagement du territoire de 1950 à 1985, Paris, La Documentation française, pp. 34-35, 1985.

10 G. Marcou, “La loi sur le Grand Paris: le retour de l’État aménageur ?”, $A J D A, \mathrm{n}^{\circ} 33 / 2010,11$ octu60 bre, pp. 1868-1877. 
Aunque la región sea de creación reciente, mientras que el departamento se remonta a la Revolución francesa, tienen en común el haber sido creados por las necesidades del Estado, a diferencia del municipio. Recordemos que según la Constitución de 1791 el departamento formaba parte de la "administración interior del reino", subordinado al poder ejecutivo. La región, como circunscripción administrativa, apareció en los años 50 para la puesta en práctica de la política de desarrollo regional del Estado. Esta característica común está encarnada de forma duradera en la institución del prefecto. Tanto el departamento como la región han sido en primer lugar elementos del poder central antes de convertirse además en la sede de una entidad territorial.

Pero existe también una diferencia. Aunque los departamentos hayan arraigado con el tiempo, su delimitación es el fruto de un compromiso que proviene en gran medida de una visión racionalista y abstracta del territorio; la soberanía nacional es una y es indiferente a las particularidades territoriales. Al contrario, la región procede de una visión geográfica sensible a las diferencias que caracterizan un territorio y que la política económica no puede ignorar. Esta visión explica también la ambigüedad de la región. Ha recogido, durante el siglo XIX y a comienzos del XX, la oposición a los departamentos de los nostálgicos de las provincias "tradicionales" del Antiguo Régimen sin que, sin embargo, los regionalismos hayan llegado nunca a imponerse como fuerzas políticas significativas. Pero el regionalismo, como componente de una ideología tradicionalista de la que Charles Maurras ha sido en su tiempo el representante más señalado, ha influenciado después en las corrientes políticas y culturales más diversas, del petanismo ${ }^{11}$ al socialismo ${ }^{12}$, pasando por el discurso del primer ministro Jean-Pierre Raffarin el 17 de marzo de 2003 ante el Congreso ${ }^{13}$, y el mismo Informe Balladur que se hizo eco en su introducción (pp. 7-8). A propósito de las regiones se refiere a las "identidades", para oponerse a las divisiones administrativas que no las reflejarían, o por el contrario poner en valor las identidades colectivas. Se insinúa así que la pertenencia a un territorio es un elemento de la ciudadanía, lo que excluye en principio la concepción francesa de la soberanía nacional, pero que consagran, en una cierta medida, las nuevas disposiciones introducidas por la ley de 16 de diciembre de 2010 (art. 26 a 29) tendentes a favorecer la fusión de regiones o de departamentos, o de una región con sus departamentos o la modificación del vínculo regional de un departamento, subordinando la decisión de la puesta en práctica del proyecto al "acuerdo" de la mayoría absoluta de los sufragios expresados por los electores en un referéndum en el cual habría participado al menos un cuarto de los electores inscritos en el departamento o en la región, según el caso.

11 Vers la révolution communautaire. Les Journées du Mont-Dore, 10-14 abril 1943, Paris, Sequana.

12 Ver el libro de Michel Vauzelle, La France déroutée. Pour des régions politiques, La Tour d'Aigues, éd. de l'Aube, coll. "Mondes en cours", 2002: el autor habla de su deseo de que las "regiones políticas" sean "pequeñas patrias" (p. 73), idea tomada de Maurras (De la politique naturelle au nationalisme intégral, textos seleccionados por F. Natter y C. Rousseau, Paris, Vrin, 1972, pp. 129s.). También hay que citar a Robert Laffont, La révolution régionaliste, Paris, Gallimard, 1967 y a algunos otros.

13 http://www.assemblée-nationale.fr/12/congres/cra/cra-01.asp 


\section{EL INFORME BALLADUR FAVORABLE A UN FORTALECIMIENTO DE LAS REGIONES}

Después de la indignación provocada por la propuesta del Informe Attali de suprimir los departamentos en diez años en beneficio de una organización basada en las regiones y las intermunicipalidades ${ }^{14}$, el Informe Balladur tuvo el cuidado de indicar que la "simplificación" de las estructuras "no implica la supresión de tal o cual nivel de administración” (p. 66). Al dilema histórico evocado más arriba, el Informe Balladur respondía que era preciso mantener los dos niveles y "fortalecer el papel de la región en el paisaje administrativo francés acercándola al departamento” (p.68). Pero la orientación que se traza es la de fortalecer las regiones francesas y el informe sugiere que sería deseable llegar a un quincena de regiones teniendo cada una al menos tres o cuatro millones de habitantes (p. 66). Para fortalecer las regiones proponía elegir "consejeros departamentales" con sistema de lista, de los cuales los primeros formarían parte también del consejo regional en función del número de votos obtenidos. El informe descartaba el mantenimiento de un escrutinio cantonal uninominal porque el elector no estaría en condiciones de efectuar él mismo la selección de los consejeros regionales (p. 70). Es por tanto esta solución la que ha sido mantenida en la ley del 16 de diciembre de 2010, inspirándose en una proposición de ley de los diputados Mancel y Bignon presentada en nombre del grupo UPM (Union pour un Mouvement Populaire), con la finalidad de confiar a los "consejeros territoriales" la administración de los departamentos y las regiones ${ }^{15}$.

\section{¿ABSORCIÓN DE LOS CONSEJOS GENERALES POR LOS CONSEJOS REGIONALES...}

La apuesta de la reforma es que a la larga el consejo regional debería acabar por absorber a los consejos generales y el procedimiento de fusión de una región con los departamentos que la componen podría ser el medio de promover esa evolución a partir de algunas regiones, pero que necesita una ley, según el artículo 72 de la Constitución, puesto que se trataría de crear entonces una nueva entidad territorial. Por ahora, y si se hace abstracción de los cálculos electorales, se espera del consejero territorial a la vez economías y una mejor coordinación de la acción pública en el cuadro regional. Las economías resultarían de la división por dos (de hecho un poco menos) del número de los electos regionales y departamentales (alrededor de 3.400 en lugar de más de 6.000) y de un mejor control de las cofinanciaciones, puesto que los mismos electos tendrían que pronunciarse sobre las subvenciones acordadas por la región y por su departamento. Existe pues un vínculo estrecho entre la reglamentación de competencias (v. infra) y la reforma de las estructuras que radica en la institución del consejero territorial.

14 Rapport de la Commission pour la libération de la croissance française: 300 décisions pour changer la France, bajo la presidencia de Jacques Attali, Paris, La Documentation Française, 2008, “decisiones” 258 a 260.

15 Proposition de loi visant à confier à des conseillers territoriaux l'administration des départements et des régions, Doc. AN sesión ordinaria 2007-2008, nº55. 


\section{... O CANTONALIZACIÓN DE LOS CONSEJOS REGIONALES?}

Sin embargo siendo elegidos los consejeros territoriales, como los consejeros generales actuales, en el marco del cantón, la reforma tendrá en primer lugar como efecto la supresión de la elección del consejo regional. Son de hecho los consejeros generales elegidos en los cantones un poco ampliados los que van a formar los consejos regionales. Desde ese momento, el impacto de la reforma es bien incierto. La reforma podría fortalecer una vez más el departamento, en la medida en que el modo de elección de los consejeros territoriales favorecerá las lógicas departamentales.

En los debates que han precedido a la reforma a menudo se ha invocado el argumento de las "parejas" para expresar lo que sería su lógica subyacente. Existiría una suerte de solidaridad funcional entre la región y el departamento, como entre el municipio y la intermunicipalidad. Este argumento justificaría la aproximación del departamento y de la región y la perspectiva de la absorción del departamento por la región. Ahora bien, no es cierto que las funciones del departamento sostengan esta interpretación. El informe de la misión de información Bélot estima por el contrario que la región y el departamento tienen "vocaciones" diferentes. El departamento es el "nivel de las solidaridades sociales y territoriales", mientras que la región es "el escalón de las misiones estratégicas y de preparación del futuro" ${ }^{\prime 6}$. El cuadro muy detallado de las competencias de los departamentos y de las regiones, que figura en el estudio de impacto del proyecto de ley, corrobora esta apreciación. La acción de los departamentos está mucho más orientada hacia lo local que hacia el espacio regional. Si es preciso hablar de una pareja, se trata más bien de una pareja entre el departamento y el bloque municipal (municipios e intermunicipalidades). Las dificultades para concebir el futuro del departamento y de la región provienen así del hecho de que el papel del nivel municipal debe ser redefinido y no lo ha sido.

\section{LA REGLAMENTACIÓN DE LAS COMPETENCIAS}

\section{Cláusula general de competencia y cofinanciaciones}

Desde la reforma de la descentralización de 1982-1986, la "clarificación de las competencias" se ha convertido en un tema recurrente de debate y de propuestas. Con el aumento de las competencias de las entidades territoriales, consecuencia de los progresos de la descentralización, "no se sabe quién hace qué". La "clarificación de las competencias" permitiría convertir a la organización administrativa en más "legible" para los ciudadanos y a los electos en más "responsables", ya que la imputación de las decisiones sería más clara. Por el contrario, la situación actual se caracteriza por la multiplicación de proyectos que afectan a varias entidades, al Estado, y a

16 Y. Krattinger et J. Gourault, Rapport d'information fait au nom de la mission temporaire sur l'organisation et l'évolution des collectivités territoriales, Sénat, n 471, 17 juin 2009, pp. 61et 63. 
veces a otras instituciones, como las Cámaras de Comercio y de Industria, sin hablar de las financiaciones europeas, lo que se acompaña de una dilución de las responsabilidades, de la multiplicación de los costes administrativos de funcionamiento para cada entidad afectada, de retrasos en la ejecución y en ocasiones de duplicidades.

En 1982, se habían anunciado "bloques de competencias", lo que habría limitado las interferencias entre entidades territoriales de diferentes niveles y con el Estado. En realidad, este principio no ha sido nunca puesto en práctica y sin duda no podía serlo: en los grandes dominios de política pública, es difícilmente imaginable que el Estado renuncie a sus responsabilidades y la delimitación de los "bloques" sería necesariamente problemática.

La ley de 16 de diciembre de 2010 afronta a su vez este problema de dos formas: por una parte propone suprimir la cláusula general de competencia de los departamentos y de las regiones y, por otra parte, establece el principio de que, al margen de las excepciones que prevé, las competencias atribuidas por la ley a una categoría de entidades territoriales lo son a titulo exclusivo, lo que prohíbe a las entidades territoriales de otra categoría intervenir en esas materias. A estos principios, la ley asocia las disposiciones tendentes a limitar las "cofinanciaciones".

\section{... UNA DESAPARICIÓN POCO PROBABLE}

Sin embargo, se puede preguntar quién quiere realmente poner fin a estas prácticas. Sin duda alguna el ministerio de Finanzas en una lógica de control del gasto público. Pero para los diferentes actores, tomados individualmente, y para el Estado, tomado bajo la figura de sus otras administraciones, las ayudas de financiación presentan numerosas ventajas. Debe tenerse en cuenta que la ley excluye, por otra parte, de las prohibiciones que enuncia a las cooperaciones entre el Estado y las entidades territoriales, y especialmente los contratos de proyecto. Para los electos locales, el lado positivo de la dilución de las responsabilidades es la mutualización de los riesgos (no solamente financieros sino también y sobre todo políticos). Las ayudas de financiación permiten también la realización de proyectos que una entidad no podría realizar aisladamente. Se aborda aquí el problema de las estructuras: si las entidades territoriales - de hecho los municipios y las intermunicipalidades- fueran de mayor tamaño, podrían solas asumir tales proyectos, con costes administrativos menos elevados. Es decir, que sin una reforma real de las estructuras es inútil reglamentar las competencias: los problemas a resolver generarán las vías de escape necesarias.

Por otra parte, numerosos organismos, que han sido creados para la puesta en práctica de políticas públicas en las que participan las entidades territoriales, dependen de las cofinanciaciones. Tal es el caso de los organismos de viviendas sociales, de múltiples organismos de estatuto asociativo o público que son los operadores de las 64 políticas sociales, en particular, de los transportes públicos. 
El gobierno había anunciado una ley sobre las competencias. De hecho el proyecto parece abandonado, pues no hay ninguna posibilidad de que pueda llegar al Parlamento antes de las próximas elecciones presidenciales y legislativas. En lugar de esto la ley introduce una reglamentación del ejercicio de las competencias, especialmente en materia de subvenciones, en sus artículos 73 a 78. Pero las disposiciones de esta reglamentación tropiezan con serios obstáculos.

Cuando la ley prohíbe en lo sucesivo a los consejos regionales y a los consejos generales deliberar sobre materias distintas a aquellas sobre las cuales la ley les da competencias, permite al mismo tiempo derogar esta prohibición "por deliberación especialmente motivada" para "todo objeto de interés regional [en su caso departamental] para el cual la ley no ha dado competencia a ninguna otra persona pública"lo que no es otra cosa que la definición comúnmente admitida de la "cláusula general de competencia". El Consejo Constitucional rechaza por razón de la misma disposición "la reclamación basada en que las disposiciones criticadas serían contrarias al principio de libre administración de las entidades territoriales" y por tanto inconstitucionales (c.55); ;al mismo tiempo constitucionaliza la cláusula general de competencia vinculándola al principio de libre administración;

Por último, si en el art. 1111-4 (al.2) del Código general de las entidades territoriales las competencias atribuidas por la ley son a titulo exclusivo a excepción del turismo, de la cultura y del deporte que son definidas como materias de competencia compartida, el mismo texto introduce una excepción mucho más grave: "No obstante, la ley puede, a título excepcional, prever que una competencia sea compartida entre varias categorías de entidades territoriales". Ahora bien, basta con abrir los códigos, por ejemplo el código de la construcción y de la vivienda o el código de la ayuda social y de las familias, para darse cuenta que la ley ha recurrido sin cesar a tales repartos, justamente para facilitar la participación de las entidades territoriales y de sus financiaciones en la puesta en marcha de las políticas públicas. Los parlamentarios eran conscientes de esa dificultad ya que introdujeron los esquemas regionales de organización de las competencias y de mutualización de los servicios, cuya adopción por la región y los departamentos permitirá el mantenimiento de las cofinanciaciones (art. 75 y 77 de la ley). Así, para poner en marcha el proyecto de especialización de las competencias que inspira estas disposiciones de la ley de 16 de diciembre de 2010, sería preciso proceder a una revisión de todos los códigos y concebir a la vez una organización de las políticas públicas radicalmente diferente. Pero para esto sería necesario también que la reforma de las estructuras haya sido llevada a cabo.

Como se ve, la reforma de las entidades territoriales está lejos de su terminación.

La primera elección de los consejeros territoriales debe tener lugar en 2014 y la entrada en vigor de las nuevas disposiciones relativas a la reglamentación del ejercicio de las competencias será el primero de enero de 2015. Aunque la reforma sea puesta en marcha, su éxito no está garantizado. 
